\title{
Montreal Cognitive Assessment Performance in HIV/AIDS: Impact of Systemic Factors
}

\author{
Noshin Koenig, Esther Fujiwara, M. John Gill, Christopher Power
}

\begin{abstract}
Background: A large proportion of people living with human immunodeficiency virus/acquired immunodeficiency syndrome (HIV/AIDS) suffer from neurocognitive impairment (NCI). The causes of the NCI are multifold in HIV infection although a subset of HIV/AIDS patients are affected by the spectrum syndrome, HIV-associated neurocognitive disorder (HAND). We investigated the Montreal Cognitive Assessment (MoCA) in relation to clinical, demographic and laboratory findings as well as its ability to predict symptomatic HAND (sHAND) among patients with HIV/AIDS. Methods: All subjects were receiving regular HIV care including CD4+ T cell counts, plasma viral load measurements, clinical evaluations and antiretroviral therapy. The diagnosis of sHAND was based upon clinical, neuroimaging, and neuropsychological assessments. Results: Among HIV-1 seropositive subjects $(n=125)$, ethnicity, education and employment were positively correlated with their MoCA scores $(p<0.05)$. In contrast, polypharmacy, central nervous system penetration-effectiveness $(\mathrm{CPE})$ score, antiretroviral drug exposure, substance use and nucleoside/nucleotide reverse transcriptase inhibitor side effects were negatively correlated with MoCA scores $(p<0.05)$. Of note, MoCA scores were not associated with CD4 T cell nadir levels, age, peak viral load, or veterans aging cohort study index. In subjects with or without sHAND, mean MoCA scores differed (sHAND, 22.8 \pm 3.51 ; non-HAND 25.2 \pm 2.64$)(\mathrm{p}<0.05)$ with a receiver operating characteristic curve showing an area under curve of 0.71 and an optimal MoCA cut-off value of 23.5 when compared to the established diagnostic paradigm. Conclusions: MoCA scores were generally lower in this HIV/AIDS population compared to reported scores in the general population. MoCA performance was associated with multiple clinical variables but displayed limited predictive utility in detecting sHAND.
\end{abstract}

RÉSUMÉ: Résultats du Montreal Cognitive Assessment chez des patients atteints de VIH/SIDA : impact de facteurs systémiques. Contexte: Une grande proportion des individus porteurs du virus de l'immunodéficience humain/syndrome de l'immunodéficience acquise (VIH/SIDA) sont atteints de déficits neurocognitifs (DNC). Les causes des DNC sont multiples dans l'infection par le VIH, bien qu'un sous-groupe de patients atteints de VIH/SIDA présente le spectre des troubles neurocognitifs liés à l'infection par le VIH (TNCV). Nous avons examiné le Montreal Cognitive Assessment (MoCA) en lien avec les observations cliniques, démographiques et biochimiques ainsi que la capacité de ce test à prédire les TNCV symptomatique (TNCVs) chez des patients atteints de VIH/SIDA. Méthode: Tous les sujets recevaient des soins réguliers pour l'infection par le VIH incluant un décompte des cellules T CD4 +, une mesure de la charge virale, des évaluations cliniques et un traitement antirétroviral. Le diagnostic de TNCVs était basé sur la clinique, la neuroimagerie et les évaluations neuropsychologiques. Résultats: Parmi les sujets séropositifs pour le VIH-1 ( $\mathrm{n}=125)$, l'ethnicité, le niveau de scolarité et l'emploi étaient corrélés positivement aux score du MoCA $(\mathrm{p}=0,05)$. Inversement, la polypharmacie, le score d'efficacité de pénétration à travers la barrière hématoencéphalique, l'exposition à des antirétroviraux, la consommation de drogues et les effets secondaires dus à des inhibiteurs nucléosidiques/nucléotidiques de la transcriptase inverse étaient corrélés négativement aux scores du $\mathrm{MoCa}(\mathrm{p}=0,05)$. Il est à noter que les scores du MoCA n'étaient pas associés aux décomptes les plus bas de cellules T CD4 +, à l'âge, aux charges virales les plus élevées, à l'indice du Veterans Aging Cohort Study (VACS). Chez les sujets avec ou sans TNCVs, les scores moyens du MoCA étaient différents (TNCVs, 22,8 $\pm 3,51$; non-TNCV 25,2 $\pm 2,64)(\mathrm{p}=0,05)$, la courbe ROC montrant une surface sous la courbe de 0,71 et une valeur seuil optimale du MoCA de 23,5 par rapport au paradigme diagnostique établi. Conclusions : Les scores du MoCA étaient généralement plus bas dans cette population de patients porteurs du VIH/SIDA par rapport aux scores rapportés pour la population en général. Les résultats du MoCA étaient associés à de multiples variables cliniques. Cependant, ils présentaient une utilité prédictive limitée pour détecter un TNCVs.

Keywords: CNS penetration efficacy, HIV/AIDS, HIV-associated neurocognitive disorders, Montreal Cognitive Assessment doi:10.1017/cjn.2015.306

Can J Neurol Sci. 2016; 43: 157-162

Despite the widespread use of potent antiretroviral therapies (ARTs), neurocognitive impairment (NCI) has remained a serious clinical challenge for patients infected by the human immuno- deficiency virus (HIV) but NCI is especially common among persons with the acquired immunodeficiency syndrome (AIDS). ${ }^{1-3}$ The consequences of neurocognitive impairment are

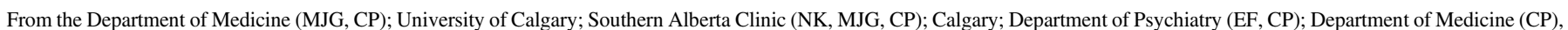
University of Alberta, Edmonton AB Canada.

Received January 8, 2015. Final Revisions Submitted June 22, 2015.

Correspondence to: Christopher Power, Department of Medicine (Neurology), University of Alberta, Heritage Medical Research Centre, 6-11, Edmonton, T6G 2S2, Canada.

Email: cp9@ualberta.ca. 
multiple including worsened adherence to therapy, obstacles in daily living and worse survival, depending on the severity of the disability. The causes of NCI in HIV/AIDS are multifactorial and encompass the direct neuropathogenic effects of the virus, resulting in HIV-associated neurocognitive disorders (HAND), as well as past or present substance abuse, traumatic brain injury (TBI), opportunistic central nervous system (CNS) infections, polypharmacy (the amount and interactions of prescribed non-HIV medications), cerebrovascular disease, hepatitis $\mathrm{C}$ virus (HCV) co-infection and possibly neurotoxic effects of some ARTs. ${ }^{2,4}$ The prevalence of HAND varies (20-50\%), depending on the study, clinical setting and assessment protocols. ${ }^{3,5-7}$ While HIVassociated dementia (the most severe form of HAND) is typically not observed during successful viral suppression, the milder forms of HAND such as minor neurocognitive disorder (MND) and asymptomatic neurocognitive impairment (ANI), often defined by disturbances in psychomotor speed, executive functions and memory, are increasingly reported as people with HIV/AIDS live longer. ${ }^{7}$ The underlying neuropathological features of HAND include chronic neuroinflammation together with synaptic loss and neuronal death. ${ }^{1}$ The impact of chronic exposure to different antiretroviral drugs has come under close scrutiny in recent years in part because of efforts to select drugs with higher brain penetration, assessed by the CNS penetration efficacy (CPE) score as well as the reports of the neurotoxic potential of some ART agents. 8,9

When screening for HAND an appropriate tool should be validated for both the detection of neurocognitive cognitive impairment and the principal predictors of HAND. The Montreal Cognitive Assessment (MoCA) is a popular screening tool for neurocognitive disorders and reliably differentiates between cognitively intact individuals and those with neurocognitive impairment in Alzheimer's disease, Parkinson's disease and vascular dementia. ${ }^{10-14}$ Both its brevity and its broad applicability make the MoCA an appealing test for identifying patients with NCI. The present study objectives were twofold; the first was to determine the relationships between the MoCA score and individual demographic, biological comorbid factors within an HIV/ AIDS cohort receiving active care and the second was to examine the sensitivity and specificity of the MoCA for symptomatic HAND in the same cohort of HIV-infected person(s).

\section{Methods ANd Materials}

\section{Study population}

The protocol including the informed consent was approved by the University of Calgary's Human Ethics Committee. Patients with cognitive complaints reported by the patient (or by his/her caregiver) from June 29, 2009 to January 06, 2014 were asked to complete the English version of the MoCA as a part of a neurocognitive assessment for determining symptomatic HAND. ${ }^{2,15,16}$ Montreal Cognitive Assessment scores and clinical data were evaluated in HIV-1 seropositive persons receiving ongoing care at the Southern Alberta Clinic (SAC) in Calgary, Alberta, Canada. Southern Alberta Clinic (SAC) provides free multidisciplinary care, including ART, for all HIV patients residing in Southern Alberta. In order to determine the predominant biological predictors of sHAND and MoCA performance, patients with NCI related to clinically proven pre-existing conditions (TBI, substance abuse, etc.) were excluded $(<5.0 \%)$. Additionally, nine patients were excluded from the initial selection, eight patients were excluded due to low educational levels ( $<$ Grade 7 ), one patient's test results was removed due to incomplete testing and serial test entries from five patients were removed and only the initial score was included in the present analyses.

\section{HAND diagnosis}

Based on the Frascati criteria there are three distinguishable categories for HAND differing in measures of dysfunction including ANI, MND and HIV-associated dementia (HAD). ${ }^{17}$ Given the small subset of patients with MND and HAD in our sample $(n=24)$ these patients were placed in a group designated as symptomatic HAND (sHAND). The diagnosis of sHAND was based on a multi-step clinical assessment, including neuropsychiatric symptoms (impairment in memory, concentration, motor functions and gait) conveyed through self-report or by the patient's caregiver/family member and verified by neuropsychological testing, neurological and medical assessments and neuroimaging. ${ }^{2,17}$ Neuropsychiatric symptoms were evaluated during clinical visits. If neurocognitive impairment was suspected, a brief neuropsychological test battery was assessed including Symbol-Digit Modalities test, Grooved Pegboard, Trails A and Trails B together with the MoCA. In addition, the patient's complete medical and social history was reviewed, which involved screening for prior neurological disorders (e.g., traumatic head injury, substance abuse) together with a physical examination, neuroimaging, and cerebrospinal fluid analyses to exclude nonHAND causes of neurocognitive impairment. Other relevant variables including education, ethnicity, substance abuse history, Hepatitis C Virus serostatus, CD4 T cell levels, plasma HIV-1 viral level, antiretroviral regimen, veterans aging cohort study (VACS) index and quality of life measurement ${ }^{22}$ at the time of testing were analysed. The diagnosis of SHAND was determined based on the collective assessments by the SAC clinical team.

\section{MoCA description}

The MoCA is a well-known neuropsychological screening tool available in the public domain (www.MoCAtest.org). ${ }^{18}$ It consists of cognitive subtests in areas of; executive functioning (assessed by alternating trail making test, phonemic fluency and a two-item abstraction task); visuospatial ability (evaluated by a clock-drawing task and three-dimensional cube copy); language (assessed by sentence repetition, phonemic fluency and a naming task); orientation (assessed through correct identification of the current date, location, and city; short term memory (assessed through a five minute delayed recall); four subtests are used to assess attention/working memory: digit spans forward and backward, tapping test and a serial seven subtraction task. The MoCA was implemented by a trained health care provider within the clinic.

\section{Statistical analyses}

All statistical analyses were performed using SPSS (v. 21). Univariate statistics were carried out using the Student $t$ and Mann-Whitney $U$ tests to test differences between individuals with and without $s$ HAND (2-tailed). Montreal Cognitive Assessment subtest differences between SHAND and nonHAND patients were assessed with a repeated measures analysis of variance (ANOVA). In addition, Pearson/Spearman correlations were used 
Table 1: Sociodemographic and clinical features of HIV/AIDS cohort

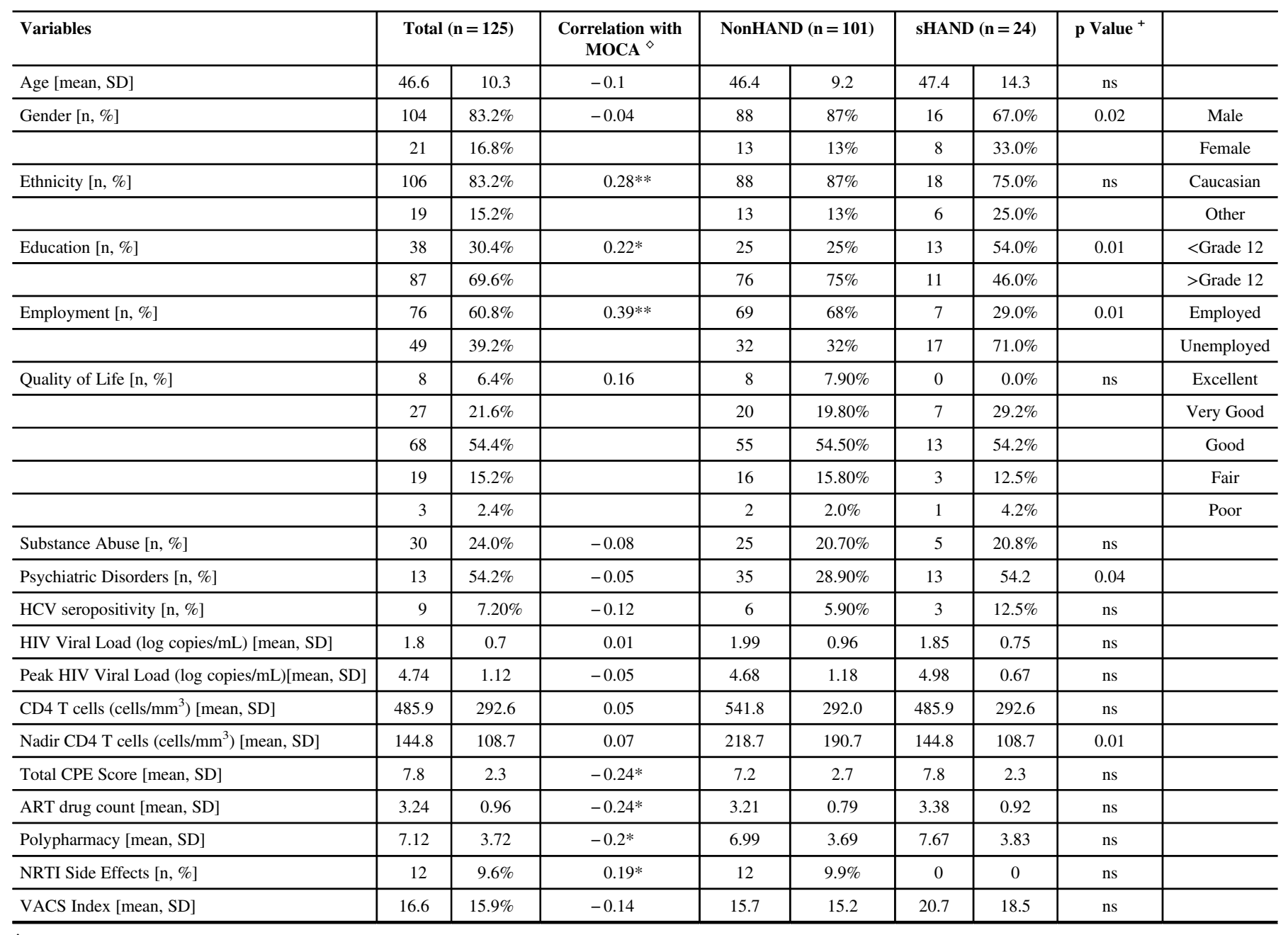

${ }^{\diamond}$ Pearson $\mathrm{r}$ for continuous variables, Spearman rho for non-normally distributed variables, or point-biserial $\mathrm{r}$ for dichotomous variables; * $\mathrm{p}<0.05$ level ( 2 tailed $) ; * \mathrm{p}<0.01$ (2-tailed); ns = not significant

${ }^{+}$Student $t$ test, Mann Whitney $U$ test and Chi-Squared test were used to evaluate comparisons of normally distributed, non-normally distributed and proportional data respectively. $\mathrm{HIV}=$ human immunodeficiency virus; $\mathrm{SD}=$ standard deviation, $\mathrm{HCV}=$ hepatitis $\mathrm{C}$ virus, $\mathrm{CPE}=$ central nervous system penetration-effectiveness, ART = antiretroviral therapies, NRTI = Nucleoside reverse transcriptase inhibitor; VACS = Veterans Aging Cohort Study;

NonHAND = non HIV-associated neurocognitive disorder; sHAND-symptomatic HIV-associated neurocognitive disorder

to test relationships between patient characteristics and MOCA scores. Point-biserial correlations were conducted between MOCA total score and dichotomous variables (e.g., gender, ethnicity). To assess the variables which contributed most to prediction of the MoCA score, multivariate linear regression was applied using only significant predictors of the MoCA previously identified in the univariate analyses. Dichotomous variables included in the model were dummy-coded. Finally, a receiver operating characteristic (ROC) curve was calculated using the MoCA-score to predict sHAND status.

\section{Results}

\section{MoCA score and clinical-demographic factors}

Neurocognitive performance represents a complex set of abilities that is influenced by multiple factors. To determine which variables were associated with MoCA scores, correlational analyses were made between the individual's MoCA and routine clinical and demographic data collected as part of clinical care. Among all subjects in the present study $(n=125)$, the mean MoCA score was $24.8 \pm 2.97$. Subsequent analyses revealed that ethnicity, education and employment status were positively (and significantly) associated with total MoCA score (Table 1). However, polypharmacy, nucleoside/nucleotide reverse transcriptase inhibitor (NRTI) side-effects, total CPE score and ART counts at the time of testing were negatively (and significantly) associated with MoCA performance. Of note, many variables previously reported to be predictive of neurocognitive impairment including immune and viral burden status, age, substance abuse, concurrent psychiatric diagnosis and VACS Index did not show a relationship with the MoCA score. To explore this issue further, a multivariate regression (automatic linear model) was 
Table 2: Multivariate regression of MoCA score showing significant predictors

\begin{tabular}{l|c|c|c|c|c|c}
\hline Predictor & Coefficient & Standard Error & t & df & F & p Value* \\
\hline Employment & -21.8 & 5.96 & -3.66 & 1 & 13.38 & 0.000 \\
\hline Ethnicity & -23.1 & 7.89 & -2.93 & 1 & 8.59 \\
\hline CPE Score & -2.85 & 1.67 & -2.67 & 1 & 0.004 \\
\hline
\end{tabular}

Adjusted $r^{2}=0.268$

performed on the total MoCA score. Predictors were restricted to those previously associated with the total MoCA score in the univariate correlations. The regression model revealed that the MoCA score was best predicted by a combination of employment (dummycoded: $\quad$ unemployed $=0$, employed $=1 ; \quad \beta=21.8, \quad \mathrm{SE}=5.96$, $\mathrm{p}<0.000$ ), ethnicity (dummy-coded: Caucasian $=0$, nonCaucasian $=1 ; \beta=23.1, \mathrm{SE}=7.89, \mathrm{p}<0.004)$, and $\mathrm{CPE}$ value $(\beta=-2.85$,

$\mathrm{SE}=1.67, \mathrm{p}<0.009$ ) (Table 2). In our cohort, individuals with the highest MoCA scores had a Caucasian ethnic background, were employed, and had a low CPE score. These findings highlighted the overall low MoCA scores in this population and the susceptibility of an individual's performance on the MoCA to the effects of a wide range of clinical and demographic factors.

\section{MoCA identification of SHAND}

Among subjects diagnosed with or without sHAND, differences in gender, education, employment status, the presence of psychiatric disorder(s), and mean CD4 T cell nadir levels were noted (Table 1). The mean MoCA score was significantly lower in subjects with sHAND compared to those without sHAND (sHAND, $22.8 \pm 3.51$; nonHAND $25.2 \pm 2.64)(\mathrm{p}<0.05)$ (Table 1). Significantly different mean scores for several MoCA subtests including those in the domains of naming, orientation and delayed recall with consistently worsened performance were seen in the sHAND group versus non sHAND patients (Figure 1A). Stratification of all individual subtests also revealed additional between-group differences in language fluency (sHAND, $0.5 \pm 0.51$; nonHAND $0.72 \pm 0.47)(\mathrm{p}<0.05)$ and attention ("tap at A task" sHAND, $0.96 \pm 0.2$; nonHAND $0.76 \pm 0.43(\mathrm{p}<0.05)$; "serial subtraction" sHAND, $1.92 \pm 0.97$; nonHAND 2.41 \pm 0.86 ) $(\mathrm{p}<0.05)$ (Supplementary Table 1). The ROC curve on MoCA scores differentiating subjects with the diagnoses of sHAND from nonHAND subjects displayed an area under the curve of 0.71 (Figure 1B). The optimized MoCA cut-off score for distinguishing sHAND from nonHAND in the present cohort was 23.5 with corresponding sensitivity $(75.2 \%)$, specificity $(62.5 \%)$, positive predictive $(37.5 \%)$ and negative predictive $(89.4 \%)$ values. These findings emphasize the limits of the MoCA in identifying subjects at risk for sHAND.

\section{Conclusions}

Although the MoCA is widely used as a screening tool for different neurocognitive disorders, the present study represents one of a few evaluations of its utility in HIV infection. Ethnicity, employment status and total CPE score were strongly predictive of MoCA performance but other factors including polypharmacy, ART drug numbers and NRTI side effects were significant, albeit negative contributors. Earlier studies have assessed the MoCA's
A

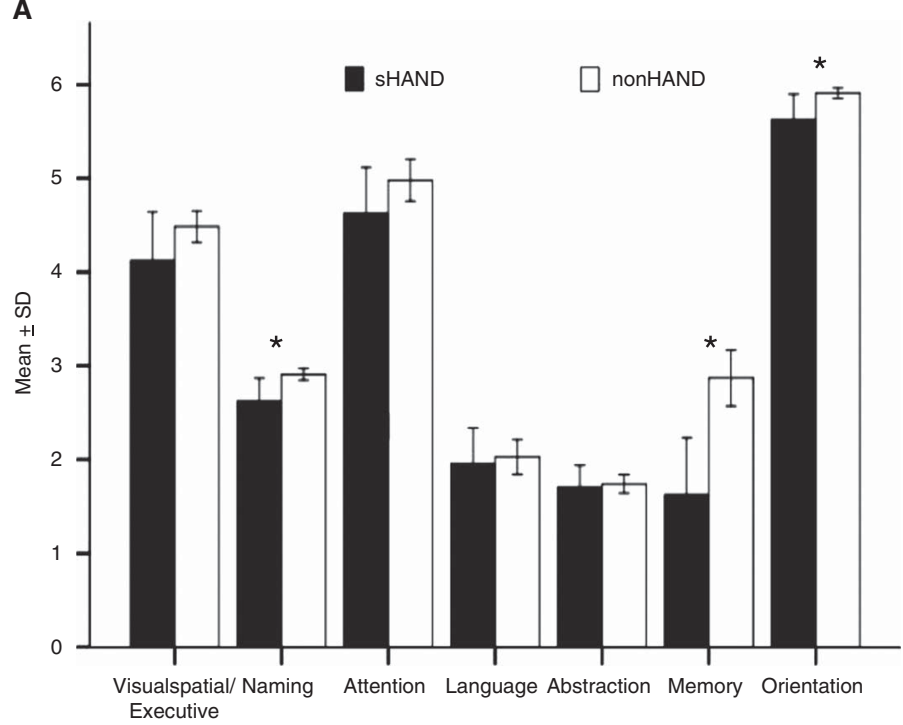

B

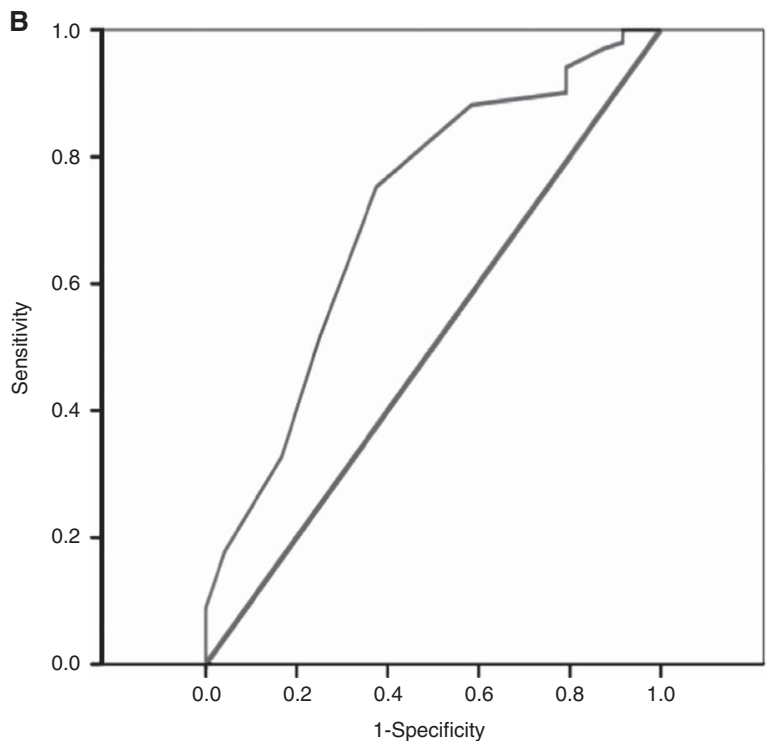

Figure 1: Analyses of MoCA performance. (A) Comparision of sHAND and nonHAND mean scores in each of the cognitive domains represented in the Montreal Cognitive Assessment. (B) Receiver operating characteristic (ROC) curve for the Montreal Cognitive Assessment. Area under the curve was noted at 0.71; 95\% CI (0.592-0.930). Cut off score of 23.5 (sensitivity $=0.752 ; 1$-specificity $=0.375)$ 
efficacy in identifying patients at risk for HAND; ${ }^{12,19}$ we have examined in the context of patients with and without the diagnosis of sHAND, which disclosed a low area-under-the-curve value with matching low sensitivity and specificity and an optimal cut off score of 23.5. These observations underline the restrictions of using the MoCA for identifying patients with sHAND despite its robust associations with several host variables.

Despite the limits raised above, these findings raise important issues as the MoCA score was dependent on three major factors including employment, ethnicity and total CPE. A positive association between employment or ethnicity and MoCA score are plausible given the composition of SAC's clientele, $\sim 65 \%$ of whom are Caucasian homosexual males and $\sim 30 \%$ are from sub-Saharan countries, often with limited education. Nevertheless, the total CPE score contributed negatively to the MoCA performance raising the intriguing possibility that the MoCA might identify persons at greater risk for ART-induced neurotoxicity due to high CNS penetration properties of select ARTs. Antiretroviral therapy-induced neurotoxicity is an increasingly recognized issue but poorly defined clinical entity. ${ }^{20}$ Indeed, the data in this area are conflicting with reports of substantial improvement or worsening in NCI depending on the ART regimens and individual cohorts. Like previous studies, we found the MoCA displayed limited capacity to detect sHAND, likely because few neurocognitive domains were assessed by the MoCA, of which only three (delayed recall, naming and orientation) distinguished sHAND from non-sHAND subjects. ${ }^{12,19}$ Indeed, the MoCA has been applied to HIV/AIDS populations in eight separate studies with variable outcomes but in general it showed low efficacies in detecting neurocognitive impairments, especially HAND (sHAND in this study). Previous studies have not examined systemic variables to the same extent as performed in the present study. ${ }^{12,13}$ Of note, the mean MoCA score for the present cohort was 24.8, which is lower than the normative MoCA cut off value, 26.0, for impairment; this latter finding speaks to the high prevalence of neurocognitive impairment in this population but also underscores the MoCA's susceptibility to the effects of subject-specific factors.

Our study has several limitations. The small sample numbers of the clinical groups forced us to combine the MND and HAD groups and might have resulted in under-powering the predictive capacity of the MoCA as well as introducing unrecognized biases. A larger study population might also have yielded MoCA associations with previously reported risk factors for HAND including age, CD4 T cell nadir, and peak viral loads, etc. Although all of the present subjects had a brief neuropsychological assessment, a more comprehensive assessment is more desirable; despite this shortcoming, our observations are similar to previous studies with in-depth neuropsychological assessments. Another challenge in the present study is the extent to which the nonHAND group contained subjects with asymptomatic neurocognitive impairment, especially as recent studies have implied that there is a risk of progression from asymptomatic to symptomatic phases; ${ }^{21}$ again only rigorous neuropsychological assessments would delineate this number but previous studies report prevalence as high as 30-50\%, depending on the individual population and test battery. Moreover, the present study did not explicitly address memory functions or attention in its standard neuropsychological battery, which may have resulted in an underestimation of the extent of NCI in this cohort. Future studies might include the MoCA as a measure of ART neurotoxicity given the present study's findings although its utility for identifying persons at risk for HAND is not compelling at present.

\section{Disclosures}

Noshin Koenig, Esther Fujiwara, M. John Gill, and Christopher Power do not have anything to disclose.

\section{ACKNOWLEDGEMENTS}

The authors thank the staff and clients at SAC for their assistance and participation in the present study. Special thanks to Soheil Ramazani and Brenda Beckthold for their assistance in collecting clinical data. This study was supported the Canadian Institutes of Health Research Emerging Team grant (CP, MJG). CP holds a Canada Research Chair (T1) in Neurological infection and Immunity.

\section{SUPPLEMENTARY MATERIAL}

To view supplementary material for this article, please visit http://dx.doi.org/10.1017/cjn.2015.306

\section{REFERENCES}

1. Chan P, Brew BJ. HIV associated neurocognitive disorders in the modern antiviral treatment era: prevalence, characteristics, biomarkers, and effects of treatment. Current HIV/AIDS reports. 2014;11:317-24.

2. McCombe JA, Vivithanaporn P, Gill MJ, Power C. Predictors of symptomatic HIV-associated neurocognitive disorders in universal health care. HIV medicine. 2013;14:99-107.

3. Heaton RK, Clifford DB, Franklin DR Jr., et al. HIV-associated neurocognitive disorders persist in the era of potent antiretroviral therapy: CHARTER Study. Neurology. 2010;75:2087-96.

4. Devlin KN, Gongvatana A, Clark US, et al. Neurocognitive effects of HIV, hepatitis C, and substance use history. J Int Neuropsychol Soc. 2012;18:68-78.

5. Hasbun R, Eraso J, Ramireddy S, et al. Screening for Neurocognitive Impairment in HIV Individuals: The Utility of the Montreal Cognitive Assessment Test. J AIDS Clin Res. 2012;3:186.

6. Chan LG, Kandiah N, Chua A. HIV-associated neurocognitive disorders (HAND) in a South Asian population - contextual application of the 2007 criteria. BMJ. 2012;2:e000662.

7. Woods SP, Moore DJ, Weber E, Grant I. Cognitive neuropsychology of HIV-associated neurocognitive disorders. Neuropsychol Rev. 2009;19:152-68.

8. Vassallo M, Durant J, Biscay V, et al. Can high central nervous system penetrating antiretroviral regimens protect against the onset of HIV-associated neurocognitive disorders? Aids. 2014; 28:493-501.

9. Cross HM, Combrinck MI, Joska JA. HIV-associated neurocognitive disorders: antiretroviral regimen, central nervous system penetration effectiveness, and cognitive outcomes. S Afri Med J. 2013; 103:758-62.

10. Kandiah N, Zhang A, Cenina AR, Au WL, Nadkarni N, Tan LC. Montreal Cognitive Assessment for the screening and prediction of cognitive decline in early Parkinson's disease. Parkinsonism \& related disorders. 2014;20:1203-8.

11. Freitas S, Simoes MR, Alves L, Santana I. Montreal cognitive assessment: validation study for mild cognitive impairment and Alzheimer disease. Alzheimer Dis. Assoc. Disord. 2013;27:37-43.

12. Milanini B, Wendelken LA, Esmaeili-Firidouni P, Chartier M, Crouch PC, Valcour V. The Montreal cognitive assessment to screen for cognitive impairment in HIV patients older than 60 years. J Acquir Immune Defic Syndr. 2014;67:67-70.

13. Overton ET, Azad TD, Parker N, et al. The Alzheimer's disease-8 and Montreal Cognitive Assessment as screening tools for neurocognitive impairment in HIV-infected persons. J Neurovirol. 2013;19:109-16.

14. Koski L, Brouillette MJ, Lalonde R, et al. Computerized testing augments pencil-and-paper tasks in measuring HIV-associated mild cognitive impairment(*). HIV Med. 2011;12:472-80. 
15. Kim DH, Jewison DL, Milner GR, Rourke SB, Gill MJ, Power C. Neurocognitive symptoms and impairment in an HIV community clinic. Can J Neurol Soc. 2001;28:228-31.

16. Vivithanaporn P, Heo G, Gamble J, et al. Neurologic disease burden in treated HIV/AIDS predicts survival: a population-based study. Neurology. 2010;75:1150-8.

17. Antinori A, Arendt G, Becker JT, et al. Updated research nosology for HIV-associated neurocognitive disorders. Neurology. 2007; 69:1789-99.

18. Nasreddine ZS, Phillips NA, Bedirian V, et al. The Montreal Cognitive Assessment, MoCA: a brief screening tool for mild cognitive impairment. J Am Geriatr Soc. 2005;53:695-9.
19. Ku NS, Lee Y, Ahn JY, et al. HIV-associated neurocognitive disorder in HIV-infected Koreans: the Korean NeuroAIDS Project. HIV Med. 2014;15:470-7.

20. Robertson K, Liner J, Meeker RB. Antiretroviral neurotoxicity. J Neurovirol. 2012;18:388-99.

21. Grant I, Franklin DR Jr., Deutsch R, et al. Asymptomatic HIV-associated neurocognitive impairment increases risk for symptomatic decline. Neurology. 2014;82:2055-62.

22. Crane HM, Van Rompaey SE, Dillingham PW, et al. A single-item measure of health-related quality-of-life for HIV-infected patients in routine clinical care. AIDS Patient Care STDs. 2006;20: 161-74. 